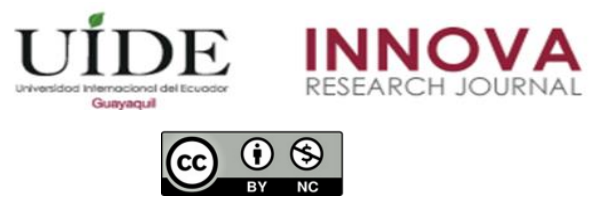

INNOVA Research Journal, ISSN 2477-9024

(Septiembre-Diciembre 2020). Vol. 5, No.3.1 pp. 216-229

DOI: https://doi.org/10.33890/innova.v5.n3.1.2020.1535

URL: http://revistas.uide.edu.ec/index.php/innova/index

Correo: innova@uide.edu.ec

\title{
Proceso de instauración institucional del acuerdo comercial entre Ecuador y la Unión Europea y su efecto en las unidades societales. Caso de estudio: sector lácteo ecuatoriano
}

\section{The Institutionalization establishment process of the trade agreement between Ecuador and the European Union and its effect on societal units. Case study: Ecuadorian dairy sector}

\author{
Santiago Francisco Carranco Paredes \\ Daniela Garcés Yépez \\ Camila Pérez Pico \\ Universidad Internacional del Ecuador
}

Autor para correspondencia: sacarrancopa@uide.edu.ec; dagarcesye@uide.edu.ec; caperezpi@uide.edu.ec

Fecha de recepción: 14 de septiembre de 2020 - Fecha de aceptación: 16 de noviembre de 2020

\begin{abstract}
Resumen
Las organizaciones internacionales son los espacios que descentralizan el sistema internacional, imparten orden, mejoran los canales de comunicación y reducen la posibilidad de conflicto. Este trabajo, se enfoca en las instituciones comerciales, específicamente en los acuerdos de libre comercio, y la forma en la que generan cambios tanto a nivel internacional como a nivel doméstico en las políticas económicas de los Estados. El objetivo de este estudio es determinar los efectos que ha generado el acuerdo comercial multipartes firmado por el Ecuador y la Unión Europea sobre un sector productivo sensible de Ecuador como es la industria de lácteos. De esta manera se estudió el proceso de institucionalización del mencionado acuerdo comercial por medio de documentación. Considerando de manera especial las estrategias planteadas para la implementación del acuerdo por parte del gobierno ecuatoriano y la participación de los representantes de las pequeñas industrias en las mesas de negociación del acuerdo. Por su parte, se realizó un análisis cuantitativo en el que se estudió el comportamiento de las importaciones en los periodos previos y posteriores al acuerdo comercial. De esta manera, por medio de una triangulación entre la teoría, el caso de estudio y los resultados empíricos, se permitió evidenciar que la sociedad civil y grupos empresariales ejercen un alto nivel de influencia en las decisiones gubernamentales. A su vez, se concluye que los efectos generados por el acuerdo en los pequeños productores de lácteos se materializan en, la reducción del precio de sus productos, lo que ha resultado en el abandono de la actividad.

Palabras claves: Comercio internacional; Instrumento internacional; Pequeña Industria; Ecuador; Unión Europea.
\end{abstract}




\begin{abstract}
The international organizations are the spaces that decentralize the international system, also provide order, improve communication channels, and reduce the possibility of conflict between States. This work focuses on commercial institutions, specifically in the free trade agreements, and the way in which they generate changes both internationally and domestically in the economic policies of States. The objective of this study is to determine the effects that the multiparty trade agreement signed by Ecuador and the European Union has generated on a sensitive productive sector in Ecuador such as the dairy industry. In this way, the institutionalization process of the commercial agreement was studied by means of documentation. Considering in a special way the strategies proposed for the implementation of the agreement by the Ecuadorian government and the participation of the representatives of small industries in the negotiation roundtables. Furthermore, a quantitative analysis was carried out to analyse the number of imports in the periods before and after the trade agreement. In this way, by means of a triangulation between the theory, the case study, and the empirical results, it was possible to demonstrate that civil society and business groups exert a high level of influence on government decisions. In turn, it is concluded that the effects generated by the agreement on small dairy producers materialize in the reduction of the price of their products, which has resulted in the abandonment of the activity.

Keywords: International Commerce; International instruments; Small scale industry; Ecuador; European Union.
\end{abstract}

\title{
Introducción
}

La inestabilidad hegemónica, la desconfianza en los multilaterales y la necesidad de expansión de la economía global, ha impulsado a los Estados a formalizar relaciones comerciales más estrechas con otros actores del sistema internacional. Esto con la finalidad de establecer intercambios más ventajosos y conseguir los objetivos comunes antes mencionados. En este proceso, Ecuador juntamente con los países miembros de la Comunidad Andina generaron iniciativas conjuntas por establecer líneas de comercio directas con la Unión Europea a partir del año 2009. Dicha iniciativa se vio institucionalizada en el marco del acuerdo comercial multipartes que Ecuador subscribió con el bloque de integración europeo, con el cual se pretende reducir las barreras arancelarias del comercio común.

Debido a las asimetrías existentes entre los países pertenecientes a las UE y Ecuador, es imperativo analizar los posibles efectos que se podrían generar tras la aplicación y cumplimiento de las normas comerciales planteadas en el acuerdo. En especial en sectores sensibles como lo es el sector agrícola. La Unión Europea mantiene agroindustrias que lo ubican como uno de los grandes productores de alimentos, productos agrícolas y ganaderos a nivel internacional, esto también es debido a sus políticas de protección y asistencia sus productores. Por ello, para el presente trabajo se ha seleccionado a la industria láctea como caso de estudio debido a que es una de las industrias más sensibles del país que competirá con la producción asistida europea.

Con el objetivo de identificar los efectos generados por el acuerdo comercial en la industria láctea ecuatoriana, se adoptó una metodología mixta, lo que permitirá analizar de manera cronológica los hitos más relevantes en el proceso de institucionalización, así como determinar los cambios económicos en las cifras de importaciones lácteas tras el año 2017. En concordancia con lo anterior, el presente artículo se divide en tres secciones. En la primera 
sección se da razón de los principios teóricos de las teorías positivistas de las Relaciones Internacionales enfocándose en las herramientas propuestas para el análisis del caso de estudio. En la segunda sección se realiza un análisis narrativo de los procesos de negociación y estrategias estatales que dieron paso a la institucionalización del acuerdo comercial. Finalmente, en la tercera sección se presenta un análisis cuantitativo del comportamiento de las importaciones lácteas con el fin de determinar la influencia del acuerdo comercial en la balanza comercial del país y su repercusión en las organizaciones productivas nacionales.

\section{Una mirada liberal de las relaciones globales: Interdependencia, cooperación e instituciones}

Los fenómenos y procesos que ocurren a nivel internacional como la guerra, la paz, la cooperación o los procesos de integración (entre otros), pueden ser comprendidos desde varias tradiciones ontológicas de la Política Internacional (Reus-Smit y Snidal 2010). Por ejemplo, en los diferentes casos que existen sobre la firma de acuerdos binacionales o multilaterales entre estados, pueden ser concebidos y analizados mediante una tradición realista o una tradición liberal de Relaciones Internacionales (RR. II).

La primera posición referenciada, prepondera la agencia estatal e imagina un sistema internacional anárquico. Este sistema produce que los Estados velen netamente por sus intereses nacionales y conciban a la cooperación como un juego de resultados suma cero. En estas condiciones, ningún estado puede arriesgarse a confiar en sus contrapartes, ya que cualquier nivel de interdependencia pondría en riesgo su capacidad autónoma de reacción ante amenazas a su seguridad nacional (Wohlforth 2010). En este sentido, los acuerdos binacionales o multipartes de cualquier índole quedarían relegados o sin efecto cuando estos se contrapongan al interés estatal de una de las partes contratantes, o en su defecto, serían utilizados netamente como instrumentos para imponer los intereses de una de las partes (Mearsheimer 1994). Es así como Baldwin (2016), propone que las instituciones no son otra cosa que el canalizador del poder internacional, pues un Estado puede imponer ideas o parámetros al interior de una institución con el objetivo de que otros Estados se subordinen a ellas, legitimando de esta forma una injerencia en otros estados.

\section{Figura 1}

Relación entre Estado e instituciones desde la perspectiva realista

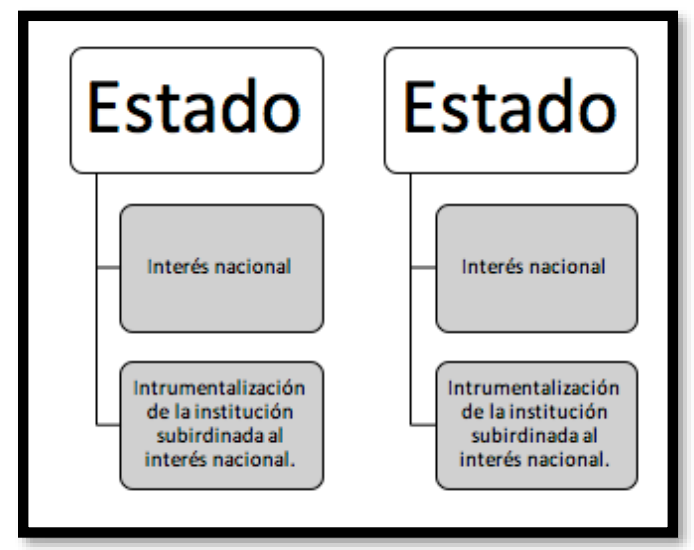

Figura elaborada por los autores. 
La posición realista, no permite analizar los efectos comerciales producidos por un instrumento jurídico acordado entre dos o varias potencias estatales, ya que su análisis se centra netamente en dos niveles, uno sistémico y uno estatal. En este sentido, los instrumentos, organismos o instituciones, no son concebidos como actores agenciales dentro de los procesos a nivel global. Por esta razón, el acuerdo de libre comercio entre Ecuador y la UE según la visión realista no es más que una herramienta utilizada por los estados con mayor poderío para difundir sus ideas y cumplir sus intereses nacionales a través de ellas. Para efectos del presente estudio, esta perspectiva teórica no sería útil debido tres motivos. En primer lugar, el realismo no daría paso al análisis del rol de la sociedad civil como un actor en la política internacional, por lo que se omitirían grandes partes del proceso de negociación, si se partiera de dicha teoría. En segundo lugar, no sería posible mantener un análisis del efecto del acuerdo sobre la política exterior ecuatoriana, ya que, según el realismo, este no tendría por qué afectar las directrices generales de la misma. También, se eliminaría la posibilidad de concebir a la institución (acuerdo de libre comercio) como un espacio de cooperación internacional en el que se genera beneficios mutuos tanto para el Ecuador como para la Unión Europea.

Por su parte, la tradición liberal de RR. II, amplía el rango de los posibles actores que pueden mantener una injerencia a un nivel global. A su vez, uno de los principales enunciados de la teoría liberal, el cual se encuentra en discordancia con el realismo, es que, si bien concuerda que el sistema internacional se encuentra en un estado de anarquía, esto no significa que los interactuantes mantienen un constante estado de conflicto y peligro, ya que, existen patrones, dinámicas e instituciones que generan un orden en las prácticas, expectativas y procesos en un nivel que se extrapola de lo doméstico. Según Dunne (2001), las organizaciones e instituciones internacionales son los espacios que descentralizan el sistema internacional, imparten orden, mejoran los canales y reducen la posibilidad de conflicto. Si bien para esta visión teórica los Estados siguen siendo un elemento de análisis debido a su rol fundamental en la política internacional, otros entes también son considerados como actores debido a su influencia directa o indirecta en el contexto internacional. Entre ellos se encuentran las organizaciones internacionales, las corporaciones multinacionales y la sociedad civil (Barnett y Sikkink 2010).

\section{Figura 2}

Relación entre Estado e instituciones desde la perspectiva liberal

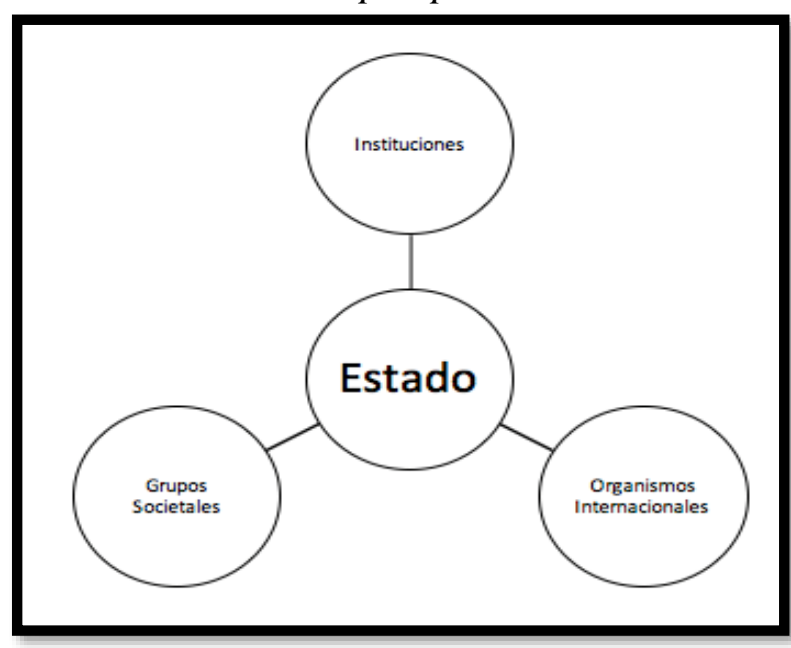

Figura elaborada por los autores. 
Desde esta perspectiva, el presente estudio toma sentido, ya que la vertiente liberal institucional, propone que los acuerdos multipartes o binacionales, así como los organismos de integración regional como los multilaterales se comportan como instituciones internacionales que logran mantener agencia y modificar el comportamiento de los estados (Figura 2). Por su parte, se propone que la política exterior de un estado, no solamente es influenciada por el contexto internacional, ya que las unidades societales que conviven dentro de los límites territoriales de este, pueden cambiar de preferencias e influir en un cambio de la política exterior de un estado. Esto permite explicar por qué en un contexto específico el Ecuador no se unió a la firma del acuerdo comercial con la UE con sus contrapartes peruanas y colombianas, mientras que, en otro contexto, la sociedad civil presionó para que esto suceda.

\section{Interdependencia global: Anarquía organizada}

El comercio global siempre ha tenido un importante espacio dentro de la política internacional. En la actualidad se ha convertido en una práctica común entre los Estados gracias al modelo económico de producción que se mantiene como consecuencia de un constante proceso de globalización. Autores como Surugiu y Surugiu (2015), plantean que el complejo proceso de globalización en el área económica implica una mayor interdependencia entre Estados, puesto a que este se ve impulsado por el flujo comercial de intercambio de bienes y servicios que traspasan las fronteras de los territorios. El proceso de globalización e interdependencia entre países ha aumentado con el paso del tiempo, debido a que el desarrollo de la tecnología ha permitido el perfeccionamiento de nuevas operaciones logísticas de intercambio que facilitan la transportación de mercancías. De esta manera, los estados desarrollan interdependencia económica, en primer lugar, por la demanda que se genera en sus mercados de los bienes y servicios que provienen de otros territorios y que son fundamentales para su economía. Y, en segundo lugar, por la inversión monetaria y el constante flujo de capitales que se ha desarrollado entre los países.

Por ende, es posible comprender el deseo y el impulso de Ecuador para fomentar la subscripción de un acuerdo de libre comercio con la UE, pues este bloque regional "es el segundo destino más importante de las exportaciones ecuatorianas y uno de los proveedores más representativos de la maquinaria industrial que ingresa al país" (Acción Ecológica, 2015, p. 1).Las características de la globalización permiten explicar la necesidad que tiene Ecuador de establecer un mercado mutuo con la UE, pues los vínculos que generará el país gracias al acuerdo le otorgarán mayores facilidades para el intercambio comercial que es tan necesario en el mundo contemporáneo.

\section{Cooperación internacional e instituciones}

Como se mencionó en al anterior apartado, el neoliberalismo institucional es una de las ramas teóricas de las RRII que enfoca su análisis en el efecto y la influencia que tienen las instituciones en el comportamiento de los actores internacionales. Una de las características relevantes de esta teoría es el hecho de que, a pesar de que reconoce a las empresas, la sociedad civil y otros organismos como actores que participan en el sistema internacional, grandes autores como Keohane (2013), admiten que los Estados siempre han sido y seguirán siendo los actores más importantes en la política mundial. En este sentido, las instituciones lo que hacen es regular 
o modificar el comportamiento de los Estados, mas no es una variable definitoria para los mismos.

Desde la visión del neoliberalismo institucional, autores como North (1990), Keohane y Goldstein (1993) perciben a las instituciones como conjuntos de reglas o restricciones formales e informales que disponen y moldean la interacción entre diversas partes. En dicho sentido, es imperativo comprender que, para la teoría institucional, los hombres son los creadores de las instituciones, pues estas necesitan un proceso de diálogo y negociación previa para determinar si dicha cooperación en efecto es beneficiosa para sus intereses.

Debido al alcance de los conceptos, en el presente trabajo se utilizará la visión institucional en vista de que es sumamente útil a la hora de entender al acuerdo de libre comercio entre Ecuador y la UE como una institución internacional que se origina a partir de los intereses de los actores y que tiene parámetros que regularán de manera clara el intercambio comercial entre estos dos actores en un futuro. Al integrar este conocimiento al caso de estudio, se entendería que las instituciones económicas, tanto de Ecuador como de la UE influencian a la institución expresada como acuerdo de libre comercio y a la vez se ven influenciadas por las reglas que este establece.

\section{Preferencias societales y preferencias estatales}

El liberalismo prepondera la agencia de los individuos, permitiendo analizarlos desde una perspectiva de la Política Global. Stein (2009), reconoce que las instituciones internacionales generan consecuencias a nivel interno de cada uno de los Estados, pues para que una institución internacional sea verdaderamente efectiva, el acuerdo debe ser interiorizado por las instituciones políticas domésticas del actor. Así, se comprende que la relación entre instituciones políticas domésticas también afecta a las instituciones internacionales, puesto que una institución es creada únicamente cuando el conjunto de actores internos coincide en que sería beneficioso la institucionalización de un acuerdo.

Justamente Andrew Moravcsik (2012), plantea un enfoque desde las preferencias individuales para comprender la estructura internacional, enfatizando el rol de las relaciones estado-sociedad. Para desarrollar de mejor manera esta visión y vincularla directamente con el tema a tratar, debemos entender entonces, que el análisis es de abajo hacia arriba, lo que quiere decir que los individuos y grupos sociales son actores participes activos del sistema político. De esta manera, no solamente se propone analizar el efecto de la institución sobre los Estados, sino que también a los grupos societales ganadores y perdedores durante el proceso de institucionalización del acuerdo.

\section{Metodología}

El estudio se realizó en función a una metodología de investigación mixta, la misma que, utilizó una triangulación metodológica que entrelazó técnicas de recolección y sistematización de información tanto cuantitativa como cualitativa. Esto se utilizó con el objetivo de establecer los efectos de una institución (acuerdo comercial UE-Ecuador) sobre el comportamiento al interior de un Estado. Además de generar una narrativa que permitió comprender el proceso de 
institucionalización del acuerdo comercial multipartes entre el Ecuador con la UE, así como la influencia que tuvieron los actores domésticos en mutar las preferencias de política exterior del estado ecuatoriano.

\section{Técnicas de recolección de información}

La evidencia cualitativa surgió a partir de fuentes primarias emitidas por Ministerio de Comercio Exterior, mismas que fueron escritas a modo de memorias de las juntas de negociación que mantuvieron los entes gubernamentales, junto con representantes de las diferentes industrias en el Ecuador. De igual manera, el análisis de fuentes secundarias como análisis de documentos oficiales y bibliografía especializada, fueron elementos clave para comprender los procedimientos e incentivos que impulsaron al gobierno de Ecuador a suscribir el acuerdo comercial. Una de las dificultades halladas durante el desarrollo de la presente sección, fue la carencia de documentos oficiales y de la sociedad civil que narren o discutan sobre las mesas de negociación específicas de los pequeños y medianos productores de las industrias nacionales. Ya que se dio preponderancia a los sectores más tradicionales.

\section{Técnicas de sistematización de información}

La información cuantitativa se recolectó de fuentes oficiales publicadas por el MAGAP y el Banco Central del Ecuador. Con esta información, se estructuró una base de datos con 6 variables de frecuencia trimestral obteniendo 240 observaciones. El periodo de análisis corresponde a 2010 - 2019. Se consideraron las siguientes variables para el análisis: 1) PIB real per cápita; 2) Flujos comerciales totales, entendido como la suma de las exportaciones ecuatorianas a la Unión Europea y las importaciones ecuatorianas provenientes de la Unión Europea; 3) Importaciones lácteas del bloque TLCAN, que es el principal bloque de importaciones lácteas a Ecuador; 4) Importaciones lácteas provenientes de la UE; 5) Exportaciones lácteas ecuatorianas; y, 6) TLC, variable dicotómica para la presencia o no del acuerdo del libre comercio.

\section{Proceso de negociación e institucionalización del acuerdo de libre comercio entre Ecuador y la Unión Europea}

Ante la iniciativa de la Comunidad Andina y la Unión Europea de estrechar sus vínculos en materia de cooperación, en el año 2007 durante la XXI Reunión Ministerial de la CAN y la UE, se aprueba la iniciativa de dar paso al proceso de negociación de un acuerdo comercial entre los dos bloques de integración. Durante las tres rondas de negociación llevadas a cabo en este proceso, Ecuador mantuvo una postura defensiva e indecisa en cuanto a la intención de formar parte del acuerdo.

La postura del país durante los años 2007 y 2008 estuvo condicionada por la presión y rechazo existente de parte de los grupos de la sociedad civil, quienes no se encontraban conformes con la participación de Ecuador en la consolidación de un acuerdo de libre comercio. Grupos sociales como Ecuador Decide y Acción Ecológica, manifestaron su posición antiglobalización ya que temían que el acuerdo afecte de manera negativa al desarrollo de las pequeñas economías y violente diversos artículos de la entonces nueva constitución. La 
influencia ejercida por estos grupos organizados de la sociedad civil resalta la veracidad del planteamiento emitido por Diana Tussie (2015), el cual sostiene que, los actores domésticos en efecto tienen la capacidad de influir en el Estado y materializar sus intereses en las normas formalizadas a través de una institución.

De acuerdo con Alvear y Jaramillo (2017), en el año 2008 se optó por realizar negociaciones de manera individual debido a que los países miembros de la CAN presentaron una serie de asimetrías, que no les permitió llegar a un consenso sobre las formalidades con las que se llevarían a cabo las negociaciones. Si bien durante el año 2008 Ecuador llevó a cabo su acercamiento con la UE de manera bilateral, un año después se tomó la decisión de suspender las negociaciones. Dicha interrupción se dio ya que, el país consideraba prioritario la resolución del conflicto comercial relativo al banano con la Unión Europea en el marco de la OMC, mismo que fue favorable para Ecuador.

En el año 2014, Ecuador retoma el proceso de institucionalización del acuerdo comercial a través de nuevas negociaciones que se caracterizaron por el cambio de estrategias y la urgencia del país por suscribir el acuerdo. Según lo descrito por Andrade y Meza (2017) el apremio por firmar el acuerdo se debía a que en el año 2016 concluirían los beneficios brindados por el sistema generalizado de preferencias plus (SGP+). Situación que afectaría de manera negativa al país ya que se eliminarían las facilidades arancelarias para el ingreso de productos ecuatorianos al mercado europeo.

Esta nueva postura de Ecuador de retomar las negociaciones con la UE, fue criticada por diversos sectores y defendida por otros. Por un lado, grupos pertenecientes a las pequeñas y medianas empresas, así como productores agrícolas a pequeña escala se opusieron de manera radical a la firma del acuerdo comercial. De acuerdo con Falconí y Oleas (2012) este sector de la sociedad civil manifestaba su rechazo ya que el acuerdo comercial podía afectar directamente a sus ingresos y se pondría en riesgo el desarrollo endógeno del país. Por otro lado, el sector exportador ejercía presión para que el estado suscriba el acuerdo comercial en vista de que sus productos ingresarían con menores tarifas arancelarias lo cual les otorga un margen de ganancia mayor.

Lo descrito anteriormente, es un claro ejemplo de que las luchas de poder al interior de un ambiente doméstico determinarán el resultado del comportamiento estatal. Pues si bien en un inicio el país mantenía una postura indecisa sobre el acuerdo comercial debido a la presión generada por grupos activistas, una vez que los intereses de uno de los grupos económicos más importantes del país se veían afectados, el estado respondió al sector con mayor influencia. De tal manera que se llevó a cabo la subscripción del acuerdo comercial manteniendo un discurso conciliador para los sectores en oposición manifestando que "la participación de nuestro país estuvo condicionada a que se tenga en cuenta las asimetrías que existen entre la UE y Ecuador" (Villagómez, 2017).

El estado ecuatoriano administró la información correspondiente al proceso de institucionalización del acuerdo comercial de una manera extremadamente reservada. A pesar de que ya han pasado tres años de vigencia del acuerdo, hasta el día de hoy no se han transparentado la información tratada en cada una de las mesas de trabajo de cada sesión de negociación, tanto 
con las industrias nacionales como en las rondas internacionales. De acuerdo con lo manifestado por Hugo Jácome (2014) esta falta de información por parte del estado ha sido evidente no solo en temas de publicaciones, sino también en la falta de diálogo con los pequeños grupos productivos del país. La académica Tania Chicaiza (2019), sostiene que esta es una de las estrategias implementadas por el estado para limitar el conocimiento de las concesiones prometidas, con el fin de reducir el rechazo por parte de la sociedad civil, quienes en caso de contar con toda la información exigirían al estado se revalúe la firma del acuerdo comercial.

En vista de que Ecuador se encontraba negociando los términos de su adherencia a un acuerdo comercial con sus normativas institucionales ya establecidas, el estado manifestó que se puso énfasis en negociar términos de protección para salvaguardar la industria agrícola nacional. Una de las más sensibles refiere a la industria láctea de quienes sus productores primarios son personas campesinas que se dedican a esta actividad con el fin de obtener recursos extra para subsistir. Según lo publicado por el Ministerio de Comercio Exterior (2017) se evitó brindar preferencias arancelarias a productos como la leche líquida y los quesos frescos debido a que estos productos son elaborados en todas las instancias a nivel nacional.

Sin embargo, debido a la falta de información existente sobre el tema, es posible denotar que la sociedad civil dedicada a esta industria no ha estado de acuerdo debido a las constantes denuncias que los pequeños productores han realizado debido a la caída del precio y bajo consumo de la leche cruda. En virtud de que el éxito de una institución es que sea interiorizada y respaldada a nivel doméstico, el estado ecuatoriano ha tenido que implementar normativas nacionales para que la inconformidad de ciertos grupos sociales no sea un obstáculo en el cumplimiento del acuerdo comercial.

Una vez que concluyeron las negociaciones, en el año 2014 se dio inicio a un complejo proyecto diplomático con el fin de que el Protocolo de Adhesión Ecuador al acuerdo comercial multipartes sea aprobado por el Parlamento Europeo quienes tardaron alrededor de un año en emitir su decisión. A partir de ese momento, el país dio paso al proceso de aprobación doméstico durante el año 2016, para ello se implementaron medidas para la óptima aplicación del acuerdo con el objetivo de que no existieran contradicciones legales. Esta última etapa de institucionalización fue públicamente rechazada por los grupos organizados que no se encontraban de acuerdo con las negociaciones en un inicio, debido a que repudiaban la idea de que se modifique la normativa interna por dicho objetivo. Sin embargo, el país continuó con el proceso que culminó con la ratificación y depósito del acuerdo comercial en diciembre del 2016.

Como respuesta a la presión ejercida por los grupos sociales y a la vigencia del acuerdo comercial, la UE destinó diez millones de euros en cooperación no reembolsable para que se realicen programas informativos sobre el acuerdo. De igual manera, Ecuador mediante el Ministerio de Comercio Exterior realizó sesiones de socialización en diversas zonas del Ecuador para que los sectores empresariales y productivos conozcan los beneficios del acuerdo comercial. Estas acciones son de vital importancia para la sobrevivencia de una institución, puesto que el estado reconoce la relevancia que posee la sociedad civil como actor de influencia y por lo tanto es imprescindible su aceptación del acuerdo. 
Actualmente, a pesar de que el acuerdo comercial con la UE ya se encuentra institucionalizado y el país está cada vez más comprometido con el cumplimiento de sus normas; sus iniciativas de difusión no han sido asertivas. Esto se debe a que el proceso de socialización se realizó en zonas urbanas y sobre todo se difundieron los lineamientos favorables para el país, más no los compromisos hechos por el país.

Una de las principales problemáticas con el proceso de institucionalización del acuerdo comercial, fue la falta de participación de pequeños productores de las industrias más vulnerables y la reducida cantidad de información que se brindó a los productores campesinos puesto que en su mayoría no tienen conocimiento sobre la UE mucho menos sobre un acuerdo comercial.

\section{Figura 3}

\section{Hitos en el proceso de la firma del acuerdo comercial Ecuador-Unión Europea}

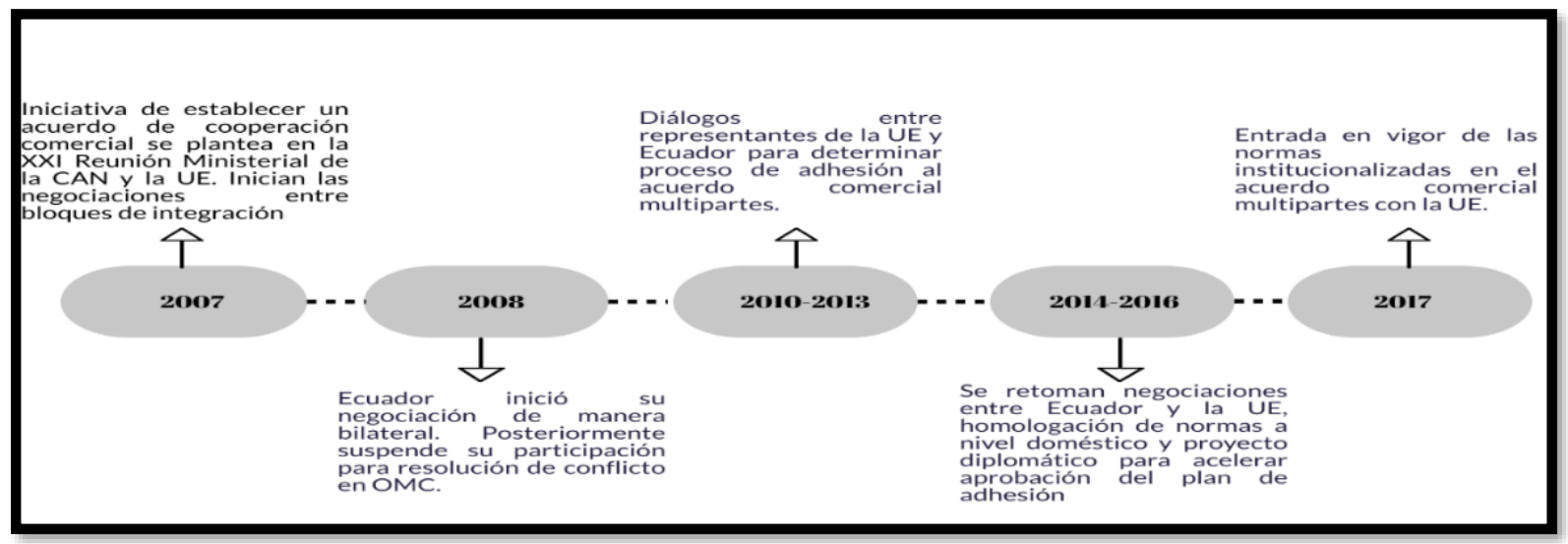

Figura elaborada por los autores.

El análisis cuantitativo presenta las estadísticas descriptivas de las variables de análisis, seguido de la presentación del modelo usado y los resultados obtenidos.

Un mercado mutuo facilita los intercambios comerciales entre las partes involucradas. Durante los diez años de análisis, el comercio entre Ecuador y la Unión Europea tan solo creció 1.33\% (BCE, 2020). Sin embargo, la tasa de crecimiento de esta variable se aceleró considerablemente tras la firma del TLC. Mientras que en el periodo 2010 - 2016 la tasa de variación promedio es de $0,95 \%$, con la firma del convenio, el crecimiento promedio de los flujos es de 2,17\%; es decir, el comercio entre las partes se duplicó tras la firma del acuerdo. A partir de 2016, Ecuador presenta un superávit comercial; tras la entrada en vigor del TLC, esta brecha ha ido decreciendo, pero sigue siendo positiva. En 2012, Ecuador registra el mayor déficit comercial. La balanza comercial entre los dos países tiene un comportamiento variable, por lo que es conveniente considerar independientemente a las importaciones ecuatorianas provenientes de la UE y a las exportaciones ecuatorianas que ingresan a territorio de la UE.

Al inicio del periodo considerando, tanto para 2010 y 2011, las importaciones registran las mayores tasas de crecimiento, $10.4 \%$ y $10.5 \%$, respectivamente (BCE, 2020). Un crecimiento 
similar se presenta en 2017, cuando las importaciones de la UE incrementan $10.2 \%$. En promedio, las importaciones tienen un crecimiento de 4,4\% tras la firma del tratado, cifra que es 2.8 veces mayor que el promedio registrado previo a la firma. Es importante mencionar que, a pesar de este incremento, en las importaciones tras la firma, todavía no se logra alcanzar los niveles de importaciones registrados en 2013, que tuvieron su pico más alto.

Por otro lado, el comportamiento de las exportaciones es diferente. Tras la firma del TLC, en 2017 las exportaciones ecuatorianas que ingresan a la UE crecen 2.6\%; en 2018 las exportaciones sufren un estancamiento e incluso para 2019 se registra un decrecimiento. La variación promedio de las exportaciones tras la firma del TLC es de $0.8 \%$. La evolución de las exportaciones previo la firma del acuerdo comercial presenta otro escenario. La tasa de crecimiento promedio es $1.4 \%$, es decir $75 \%$ mayor que el crecimiento registrado tras la firma (BCE, 2020). El año 2013 es el año con la mayor tasa de crecimiento: se registra una variación de 9,6\%. En términos nominales, el periodo con mayores exportaciones (2018, II) es tan solo $1,9 \%$ mayor que el punto más alto previo a la firma del tratado (2014, II). Es así como, el incremento de la tasa de crecimiento de las importaciones y la disminución en la tasa de crecimiento de las exportaciones reduce el superávit comercial ecuatoriano.

Ahora bien, considerando el sector de los lácteos, las exportaciones ecuatorianas se han visto severamente afectadas en la última década. El sector lácteo ecuatoriano se encuentra en déficit comercial. En los 10 años analizados, las exportaciones lácteas ecuatorianas se han reducido 96\% (MAGAP, 2020). El crecimiento promedio 2010-2016 de las exportaciones lácteas fue de 46\%; tras la firma del TLC, el crecimiento es de $89 \%$. Es importante mencionar que este promedio se ve claramente afectado por crecimientos atípicos registrados en el primer trimestre de 2017 y tercer trimestre 2019. Si consideramos valores nominales, es a partir de mediados de 2017 cuando se obtienen las cifras más bajas registradas en toda la década y esta es una tendencia que se mantiene hasta el final del periodo considerado.

Por otro lado, las importaciones lácteas provenientes de la Unión Europea han incrementado más de $50 \%$ en la última década, con un crecimiento promedio de $8 \%$ (BCE, 2020). Previo a la firma del acuerdo comercial, el crecimiento promedio de las exportaciones fue de $9 \%$, tasa que disminuye al $5 \%$ tras la entrada en vigor del tratado. Sin embargo, a pesar de esta caída, las importaciones lácteas de la UE han crecido en 54\%. Cabe mencionar que las importaciones lácteas provenientes de la UE tras la firma del acuerdo comercial no logran superar los niveles máximos alcanzados en 2011 ni en 2014.

Es importante anotar que la Unión Europea no es el único bloque que ha experimentado un incremento de sus exportaciones de productos lácteos a Ecuador. El principal socio comercial en este sector es el TLCAN, que representa $47 \%$ de las importaciones ecuatorianas. Las importaciones de lácteos de este bloque han incrementado $81 \%$ durante todo el periodo considerado; sin embargo, se han visto afectadas tras la firma del TLC con EU, las importaciones han disminuido $16 \%$. A pesar de la contracción general evidenciada tras la firma del acuerdo comercial, en términos nominales, las importaciones lácteas del bloque TLCAN registran sus valores más altos de la década en dos periodos post firma del acuerdo (Figura 4). 


\section{Figura 4}

\section{Importaciones lácteas del Ecuador}

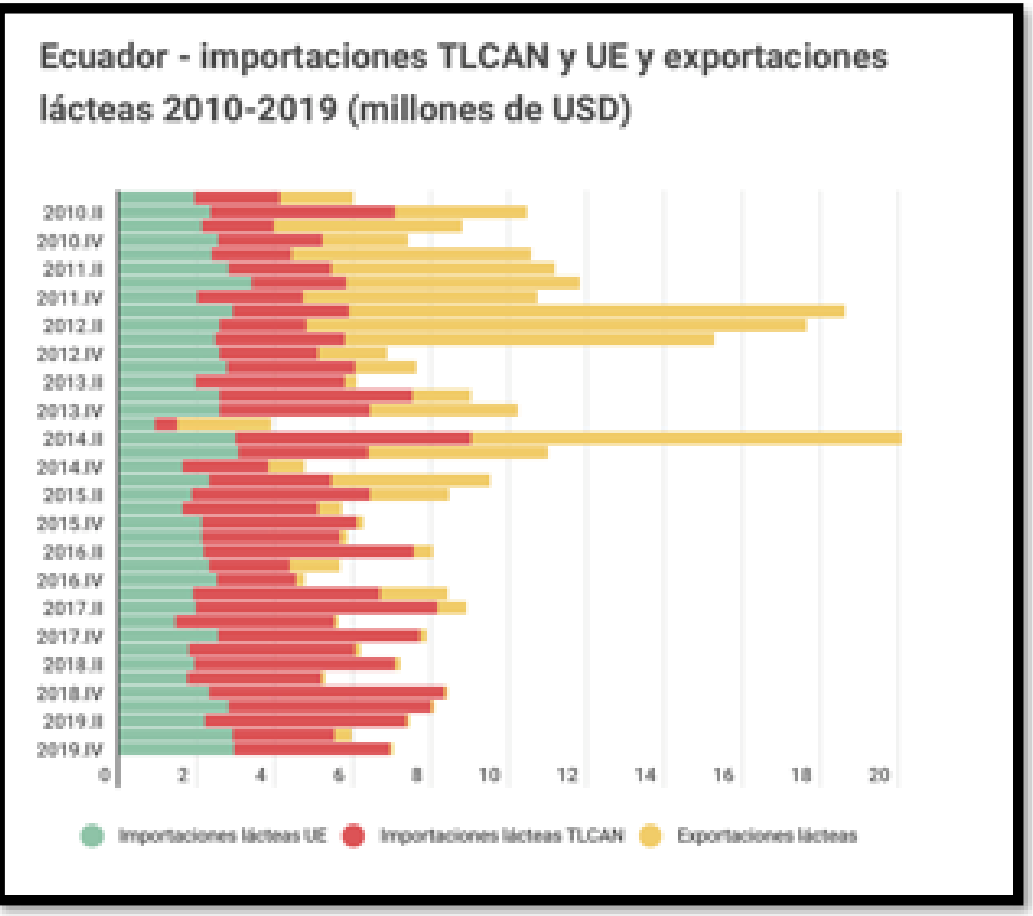

Figura elaborada por los autores a partir de los datos del BCE.

Se realizaron múltiples modelos econométricos para buscar una-relación entre las variables. Sin embargo, los modelos de regresión lineal múltiple planteados no alcanzaron significancia estadística que permita establecer relación alguna. Esta falta de significancia principalmente radica en el hecho de que el TLC fue firmado hace un corto periodo de tiempo por lo que se carece de suficiente evidencia.

\section{Conclusiones}

En función de lo planteado anteriormente, es posible evidenciar la influencia que ejercen los grupos de la sociedad civil, así como los grupos empresariales en los procesos de institucionalización del acuerdo comercial. Esto es justificado por la presión ejercida por parte de estos actores al estado ecuatoriano, con la finalidad de que la institución en construcción respalde los intereses y demandas existentes a nivel doméstico. Sin embargo, en el presente caso de estudio es claro que el estado ecuatoriano mantuvo su preponderancia como actor del sistema internacional ya que, a pesar de las demandas y oposición ejercida por grupos de la sociedad, Ecuador subscribió e implementó el acuerdo comercial con la UE. De esta manera, es posible corroborar que la creación de una institución internacional como lo es un TLC, responde a los intereses comerciales nacionales que permitan aumentar los beneficios y la rentabilidad ciertas grandes industrias nacionales a costa de los pequeños y medianos productores. 
Por su parte, la teoría es comprobada ya que en efecto una institución internacional logra modificar el comportamiento de un Estado, teniendo efectos directos en sus pequeñas industrias y en las maneras que estos se logran insertar dentro de las redes de comercio global. En caso ecuatoriano se puede observar que los efectos a corto plazo demuestran que existe un cambio en la manera de producción de los pequeños productores lecheros. En este sentido, se podría esperar ver un cambio en las actividades en ciertos espacios que tradicionalmente se han dedicado a industrias las cuales no son realmente competitivas. Futuros trabajos con respecto al tema, podrían corroborar dicha situación. El presente estudio a partir de la data obtenida ha logrado generar ciertas nociones interpretadas de dichos cambios.

\section{Agradecimientos}

Este artículo contó con el apoyo del grupo de investigación de EPI crítica de la UIDE y en especial de la estudiante Sirahí Bastidas.

\section{Referencias bibliográficas}

Acción Ecológica. (2015). Lo que Ecuador negoció con Europa. Quito: Acción Ecológica. Andrade, A y Meza, A. (2017). Acuerdo comercial entre Ecuador y la Unión Europea: El caso del sector bananero ecuatoriano. Revista Espacios, 58 (1) 1-15.

Alvear y Jaramillo. (2017). Negociaciones CAN-UE: la integración se cayó del barco. Revista La Tendencia 09, 25-50.

Baldwin, D. (2016). Power and International Relations: A Conceptual Approach. New Jersey: Princeton University Press.

Banco Central del Ecuador. (2019). Información Estadística Mensual. https://contenido.bce.fin.ec/docs.php?path=/documentos/PublicacionesNotas/Catalogo/IEMensu al/Indices/m1998082018.htm

Barnett, Michael, y Kathryn Sikkink. (2010) "From International Relations to Global Society". En The Oxford Handbook of International Relations, de Reus-Smit Christian y Duncan Snidal, 62-83. Oxford: Oxford University Press.

Cheon, D. (2010). Methods for Ex Post Economic Evaluation of Free Trade Agreements. ADB Working Paper Series on Regional Economic Integration N. 059.

Chicaiza. (2019). El conflicto lechero en Ecuador con el TLC-UE. Quito: Agencia Ecologista. Dunne, T. (2010). "Liberalism". En The Oxford Handbook of International Relations, de ReusSmit Christian y Duncan Snidal, 62-83. Oxford: Oxford University Press.

Falconí y Oleas. (2012). Implicaciones del posible Acuerdo Multipartes con la Unión Europea. Quito: FLACSO

Jácome. (2014). El retorno de las carabelas: Acuerdo Comercial Multipartes entre Ecuador y la Unión Europea. Quito: FLACSO.

Keohane, R. (2013). Institucionalismo neoliberal. Una perspectiva de la política internacional. Buenos Aires: Grupo editorial latinoamericano.

Keohane, R., y Goldstein, J. (1993). Ideas and Foreign Policy: Beliefs, Institutions, and Political Change. Cornell University Press: New York.

Mearsheimer, John J. (1994). "The False Promise of International Institutions". International Security, 5-49. 
Ministerio de Agricultura. (2020). Boletín Nacional Comercio Exterior. http://sipa.agricultura.gob.ec/index.php/boletin-nacional-comercio-exterior/2019

Ministerio de Comercio. (2017). Memorias de las Negociaciones del Acuerdo Comercial Multipartes entre Ecuador y la Unión Europea. Quito.

North, D. (1990). Institutions, Institutional Change and Economic Performance. Cambridge: Cambridge University Press.

Reus-Smit, Christian, y Duncan Snidal. The Oxford Handbook of International Relations. Oxford: The Oxford University Press, 2010.

Surugiu, M., y Surugiu, C. (2015). International Trade, Globalization and Economic Interdependence between European Countries: Implications for Businesses and Marketing Framework. Procedia Economics and Finance. 32, 131-138.

Tussie, D. (2015). Relaciones Internacionales y Economía Política Internacional: notas para el debate. Relaciones Internacionales, 24(48), 155-175

Villagómez. (2017). Acuerdo comercial multipartes Ecuador - Unión Europea. Quito: AFESE

Wohlforth, WIlliam. (2010). "Realism" En The Oxford handbook of international relations, de Christian Reus-Smit y Duncan Snidal, 131-149. Oxford: Oxford University Press 